\title{
Dinâmica espacial e temporal de zoeas de Brachyura (Crustacea, Decapoda) no estuário do Rio Jaguaribe, Itamaracá, Pernambuco, Brasil
}

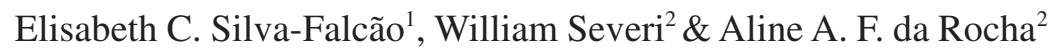

1. Departamento de Oceanografia, Universidade Federal de Pernambuco, Av. da Arquitetura, s/n, 50740-550 Cidade Universitária, Recife, PE. (elisabeth_cabral@hotmail.com)

2. Departamento de Pesca e Aqüicultura, Universidade Federal Rural de Pernambuco, Av. Dom Manoel de Medeiros, s/n, 52171-900 Dois Irmãos, Recife, PE. (wseveri@ depaq.ufrpe.br)

\begin{abstract}
Spatial and temporal dynamics of Brachyura zoeae (Crustacea, Decapoda) in the Jaguaribe river estuary, Itamaracá, Pernambuco, Brazil. A survey regarding the diel and seasonal distribution of brachyuran zoea was accomplished in the Jaguaribe estuary, state of Pernambuco, Brazil. Plankton samples were obtained bimonthly, throughout the year of 2001, in different tides and during day and night periods, at two sampling stations. Fourteen taxa were identified, among which Ocypodidae, represented by Ucides cordatus (Linnaeus, 1763) and Uca spp. zoea, was the most abundant one, followed by Xanthidae and Grapsidae. In the station close to the estuary opening, the taxa were well distributed, besides presenting a larger number of identified families. Ocypodidae, Grapsidae and Morphotype B zoea indicated a dispersion movement into coastal areas, while Pinnotheridae indicated a probable retention within the estuarine waters. The high occurrence of zoea in the first larval stages reinforces the important role of estuaries as nursery grounds for several crustacean species.
\end{abstract}

KEYWORDS. Estuaries, meroplankton, tropical.

RESUMO. Um estudo da distribuição fotoperiódica e sazonal das zoeas de Brachyura foi realizado no estuário do Rio Jaguaribe, Pernambuco, Brasil. Amostras de plâncton foram obtidas bimestralmente, durante o ano de 2001, em diferentes marés e nos períodos noturno e diurno em duas estações de coleta. Foram identificados 14 táxons, onde a família Ocypodidae, representada pelas larvas de Ucides cordatus (Linnaeus, 1763) e Uca spp., foi a mais abundante, seguida de Xanthidae e Grapsidae. Na estação próxima à foz, os táxons estiveram bem distribuídos, além de ter apresentado um maior número de famílias identificadas. As larvas de Ocypodidae, Grapsidae e Morfotipo B indicaram um movimento de dispersão para áreas costeiras, enquanto que os Pinnotheridae indicaram uma provável retenção nas águas do estuário. A elevada ocorrência de zoeas no primeiro estágio larval reforça o importante papel dos estuários como berçários para diversas espécies de crustáceos.

PALAVRAS-CHAVE. Estuários, meroplâncton, tropical.

O complexo estuarino de Itamaracá tem sido alvo de diversos estudos ambientais, sendo um dos sistemas estuarinos mais intensivamente estudados na região Nordeste do Brasil (Medeiros \& Kjerfye, 1993; VASCONCELOS-FILHO \& OliveIRA, 1999; EKAU et al., 2001; LACERDA et al., 2004; entre outros). Apesar disso, pouco se sabe sobre a ocorrência, distribuição e dinâmica do meroplâncton, especialmente dos decápodes planctônicos, neste complexo e adjacências, podendose destacar os estudos de SchwAmBorn et al. (1999, 2001).

Os estudos de dispersão e recrutamento de crustáceos decápodes têm enfatizado, principalmente, as comunidades estuarinas de zonas temperadas (WILLIAMS, 1971; SANDIFER, 1973; SHANKS et al., 2002; GonZÁLEZGordillo \& RodríGUEZ, 2003; Grabe, 2003; YANNICELli et al., 2006), tornando evidente a necessidade de estudos mais específicos acerca das comunidades meroplanctônicas estuarinas tropicais.

Impactos antrópicos nas áreas costeiras e estuarinas podem causar uma deficiência no recrutamento larval, resultando em uma baixa densidade de organismos (SILVA et al., 2004). A redução da comunidade zooplanctônica dos estuários pode promover uma considerável diminuição dos recursos pesqueiros, assim como um desequilíbrio ecológico tanto no estuário como na costa adjacente, para onde boa parte da biomassa animal e detritos do manguezal são transportados (SCHWAMBORN \& BONECKER, 1996; SCHWAMBORN et al., 1999, 2001).

O conhecimento do comportamento sazonal dessas larvas meroplanctônicas torna-se importante do ponto de vista ecológico e econômico, auxiliando em futuros projetos de manejo através da determinação da época de reprodução, fertilidade e abundância. Dentro deste contexto, o objetivo do presente estudo é analisar a distribuição temporal das zoeas de Brachyura, em um estuário da região Nordeste do Brasil, relacionando-a com parâmetros ambientais, assim como determinar sua abundância e composição taxonômica.

\section{MATERIAL E MÉTODOS}

O estuário do Rio Jaguaribe está localizado na porção nordeste da Ilha de Itamaracá, Estado de Pernambuco. Foram realizadas coletas bimestrais, durante o ano de 2001, em duas estações de coleta, uma à montante e outra próxima à foz do referido rio (Fig. 1).

Doze amostras foram obtidas através de arrastos horizontais subsuperficiais, com um barco a motor, empregando rede de plâncton com abertura de malha de $500 \mu \mathrm{m}$ nos períodos noturno (19:00-24:00h) e diurno 
(07:00-12:00h). Os arrastos tiveram duração padronizada de 10 minutos e a quantidade de água filtrada pela rede foi registrada por um fluxômetro, acoplado no centro da boca da rede. $\mathrm{O}$ material coletado foi fixado imediatamente em formol $5 \%$, neutralizado com carbonato de cálcio $\left(\mathrm{CaCO}_{3}\right)$ e acondicionado em potes de $500 \mathrm{~mL}$. Para as variáveis ambientais temperatura e salinidade, monitoradas in situ, foi utilizado um monitor portátil de campo.

Em laboratório, foi realizada a triagem das zoeas de Brachyura dos demais constituintes da amostra. Foi estimada a densidade de zoeas para cada bimestre, expressa em número de organismos por $\mathrm{m}^{3}$ A classificação taxonômica das zoeas foi realizada através de subamostras de $1 \mathrm{ml}$, retiradas da amostra diluída com o auxílio de uma pipeta automática e homogeneizadas em $100 \mathrm{ml}$ de água

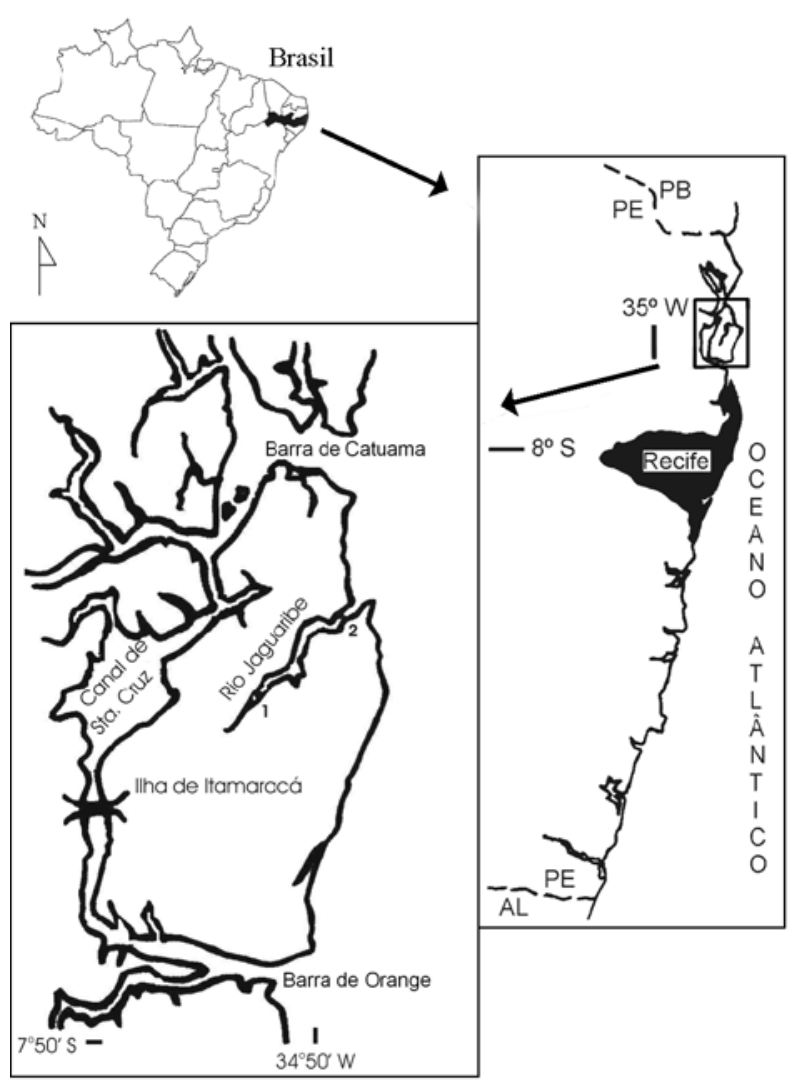

Fig. 1. Área de estudo, destacando as estações de coleta no estuário do Rio Jaguaribe, Itamaracá, PE.

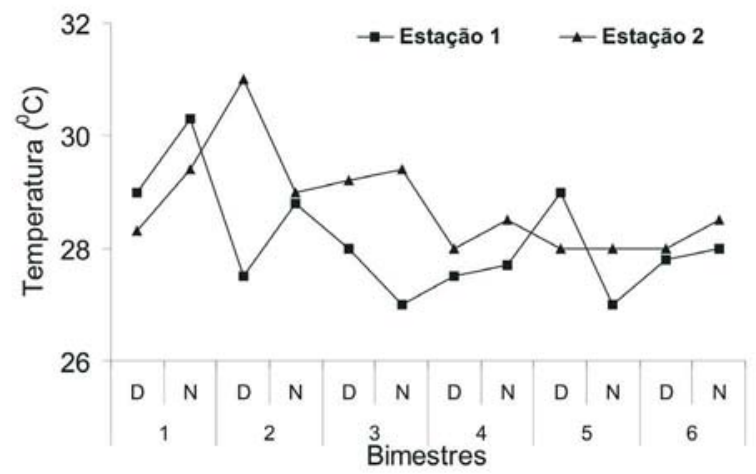

destilada. Estas subamostragens foram realizadas somente quando o número total de zoeas, triadas da amostra de zooplâncton, excedia trinta indivíduos.

A densidade total das zoeas foi calculada a partir do número total de larvas encontradas na amostra completa, enquanto que a densidade dos táxons identificados foi calculada a partir do número de indivíduos encontrados nas subamostras.

As zoeas foram identificadas em nível de família, utilizando-se a chave de PoHLE et al. (1999), e níveis específicos foram identificados através da caracterização das peças bucais, abdômen e carapaça, utilizando diversos trabalhos de descrição larval de organismos cultivados em laboratório. À medida que as zoeas foram taxonomicamente classificadas, os estágios larvais de cada uma foram determinados através da contagem de cerdas plumosas no primeiro maxilípede (PoHLE et al., 1999). Na ocasião em que não foi possível determinar a que família ou gênero pertencia a zoea, esta foi designada por morfotipo.

Para testar a significância dos fatores maré, fotoperíodo e estação de coleta sobre a densidade de organismos, foi realizado um teste não-paramétrico (Mann-Whitney), considerando significativos os valores de $p \leq 0,05$. Para a elaboração dos gráficos de densidade das diferentes espécies identificadas, foi calculada a raiz quadrada dos valores, a fim de homogeneizar os dados.

\section{RESULTADOS}

Enquanto a temperatura da água oscilou dentro de uma pequena amplitude (cerca de $3,5^{\circ} \mathrm{C}$ ), a sazonalidade da salinidade foi mais visível na estação 1 (Fig. 2), com queda dos valores a partir do segundo bimestre, se estendendo até o quarto (período chuvoso) e maiores valores do quinto ao primeiro bimestre (período seco). Nesta estação, a salinidade variou de 1,5 a 47 , e a temperatura de 27 a $30,3^{\circ} \mathrm{C}$, enquanto que, na estação 2 , esta variação foi de 13,8 a 42,5 e de 28 a $31^{\circ} \mathrm{C}$, respectivamente.

A densidade total de zoeas de Brachyura encontrada nas duas estações não diferiu significativamente, sendo que a estação 1 apresentou maior amplitude dos valores, de 0,11 a 28,91 org. $\mathrm{m}^{-3}$. Porém, os valores de densidade foram significativamente diferentes entre as marés e entre os fotoperíodos $(\mathrm{p}=0,02)$ (Fig. 3), tendo ocorrido maior densidade na maré vazante e no período noturno em ambas as estações.

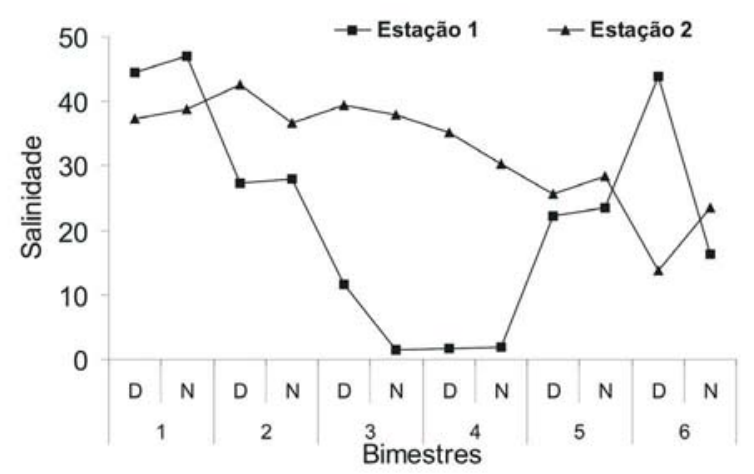

Fig. 2. Variação temporal da temperatura e da salinidade da água de sub-superfície nas estações 1 e 2 do estuário do Rio Jaguaribe, PE, durante o ano de 2001. (D, período diurno; N, período noturno). 

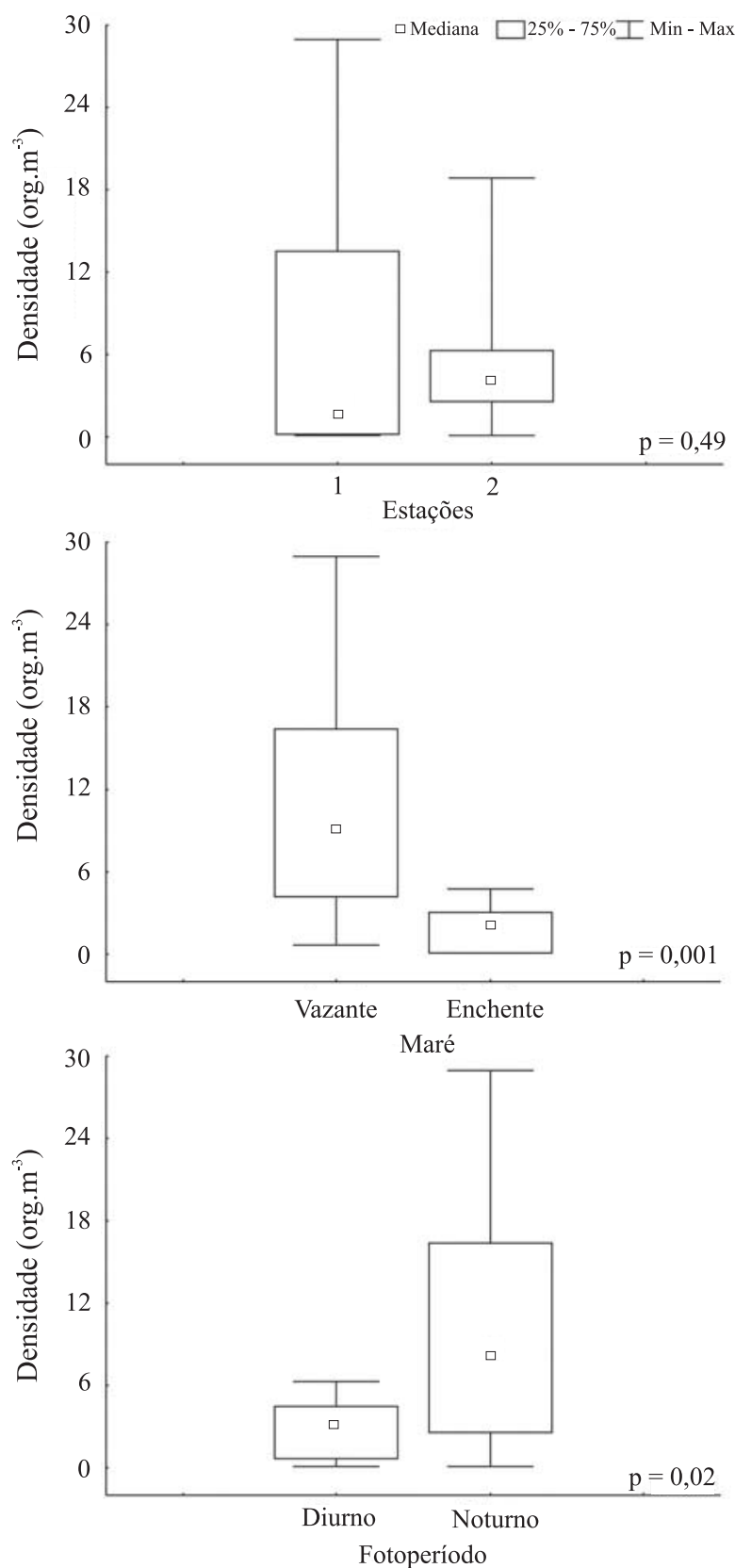

Fig. 3. Variação da densidade de zoeas de Brachyura no estuário do Rio Jaguaribe, PE, durante o ano de 2001, em função das diferentes estações de coleta, marés e condições de luz.

Ao longo do período estudado, a densidade de zoeas de Brachyura apresentou diferença significativa entre os bimestres na estação $1(\mathrm{p}=0,05)$. Nesta estação, os três últimos bimestres apresentaram maior densidade de organismos, no período noturno, com um pico no sexto bimestre, onde foi registrado um total de 28,91 org. $\mathrm{m}^{-3}$. Por outro lado, o terceiro bimestre apresentou menor densidade, com apenas 0,1 org.m ${ }^{-3}$ (Fig. 4).

Na estação 2, também houve diferença significativa na densidade entre os bimestres $(\mathrm{p}=0,01)$, tendo o quarto bimestre apresentado maior densidade no período noturno $\left(18,8\right.$ org. $\left.\mathrm{m}^{-3}\right)$.

Foram identificados 13 táxons de Brachyura (Tab. I), organizados em ordem taxonômica de acordo com MELO

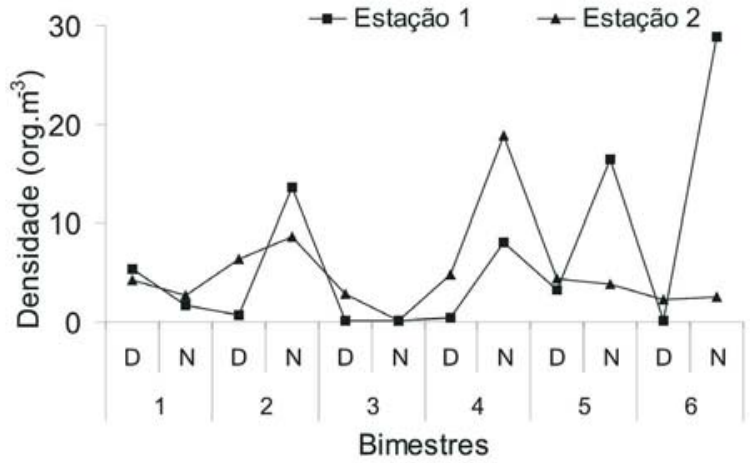

Fig. 4. Variação temporal da densidade de zoeas de Brachyura nas estações de coleta no estuário do Rio Jaguaribe, PE, ao longo de 2001 (D, período diurno; N, período noturno).

(1996). Com relação à distribuição sazonal das famílias (Fig. 5), observou-se que Ocypodidae esteve bem distribuída ao longo do ano na estação 1, predominando com mais de $50 \%$ da densidade total, nas marés vazante noturnas, exceto no quarto bimestre. As zoeas de Ucides cordatus (Linnaeus, 1763) são as principais responsáveis pela grande participação desta família, pois ocorreram durante todo o período estudado, com dois picos principais: um no período seco e outro no chuvoso. Por outro lado, as zoeas de Uca spp. estiveram presentes, nesta estação, somente no período seco e em amostras noturnas.

Os grapsídeos foram mais abundantes nos dois primeiros bimestres, destacando-se Goniopsis cruentata (Latreille, 1803) no período seco. Xanthidae também foi bem representada, presente principalmente nos dois primeiros e últimos bimestres, com predominância de Panopeus occidentalis Saussure, 1857 e Hexapanopeus caribbaeus (Stimpson, 1871). Zoeas de Xanthidae não identificadas, designadas como Outros Xanthidae, ocorreu predominantemente no quinto bimestre.

As demais famílias ocorreram em bimestres distintos do período de coleta, como Majidae, que ocorreu na vazante noturna, com um pico no período seco e outro no chuvoso. Os morfotipos A e B apresentaram maior abundância no período seco, assim como as zoeas de Pinnotheridae.

$\mathrm{Na}$ estação 2, a variação temporal das famílias apresentou-se de forma diferente, com grande participação dos morfotipos A e B, que ocorreram praticamente em todos os bimestres, porém com maior abundância no período seco. Os ocipodídeos ocorreram principalmente no período chuvoso e foram representados apenas por zoeas de $U$. cordatus. Indivíduos pertencentes a Xanthidae estiveram presentes preferencialmente no período noturno e Grapsidae foi representada por G. cruentata, no período chuvoso, e Grapsidae morfotipo A, no período seco.

Pinnotheridae apresentou uma melhor distribuição, com as zoeas de Pinnixa spp. presentes sempre na maré enchente. As famílias que ocorreram esporadicamente foram Majidae e Portunidae, no quarto bimestre, e 

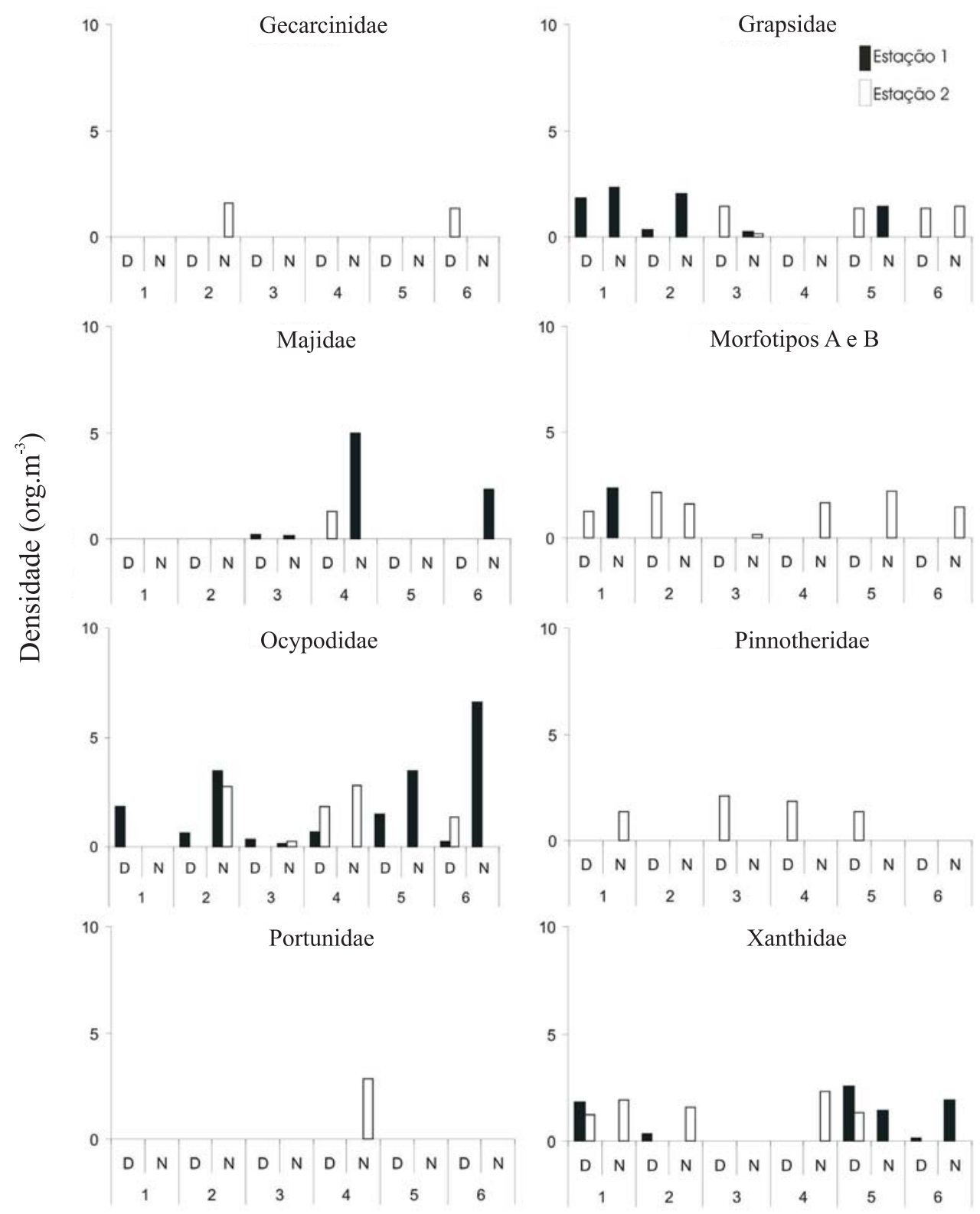

Bimestres

Bimestres

Fig. 5. Variação temporal da densidade de zoeas identificadas no estuário do Rio Jaguaribe, PE, durante 2001. (D, período diurno; N, período noturno).

Gecarcinidae, representada por Cardisoma guanhumi Latreille, 1825, que ocorreu no segundo e sexto bimestres.

Nas duas estações de coleta ocorreram os estágios larvais de zoea I a zoea IV+ (ou estágios posteriores), com predominância do primeiro estágio em ambas (Fig. 6). Pinnixa spp. foi o táxon que apresentou maior número de estágios, sendo que na estação 1 , ocorreram os estágios de zoea I a zoea III e na estação 2, ocorreram todos os estágios descritos. Ucides cordatus ocorreu nos estágios de zoea I, II e IV na estação I, enquanto que na 2, ocorreram apenas os estágios I e II. Grapsidae morfotipo A ocorreu nos estágios I e II na estação 2 do estuário em estudo. Os demais táxons ocorreram apenas no estágio de zoea I, em ambas as estações de coleta.

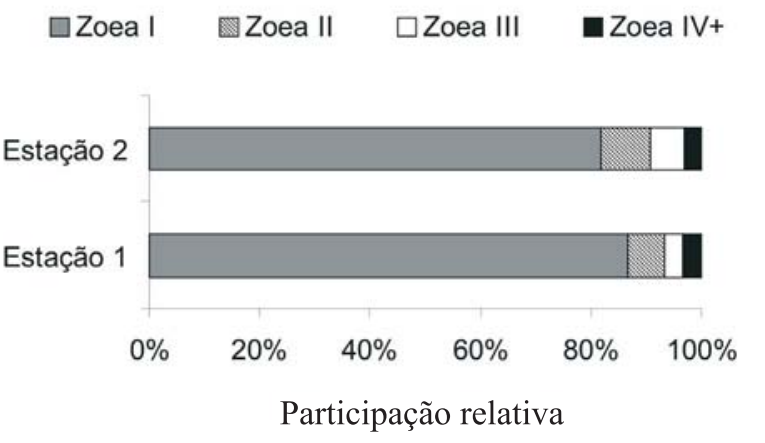

Fig. 6. Participação relativa dos estágios larvais das zoeas de Brachyura coletadas nas estações 1 e 2 do estuário do Rio Jaguaribe, PE, durante os bimestres de 2001. 
Tabela I. Lista taxonômica das zoeas de Brachyura coletadas em duas estações fixas no estuário do Rio Jaguaribe, PE, durante os bimestres de 2001

\begin{tabular}{|c|c|c|}
\hline Táxons & Período de maior abundância & Densidade relativa (org. $\mathrm{m}^{-3}$ ) \\
\hline Morfotipo A & Seco & 5,70 \\
\hline Morfotipo B & Seco & 2,98 \\
\hline \multicolumn{3}{|l|}{ XANTHIDAE } \\
\hline Hexapanopeus caribbaeus (Stimpson, 1871) & Seco & 3,40 \\
\hline Panopeus americanus Saussure, 1857 & Seco & 3,21 \\
\hline Panopeus occidentalis Saussure, 1857 & Seco & 0,02 \\
\hline Outros Xanthidae & Seco & 2,69 \\
\hline \multicolumn{3}{|l|}{ PINNOTHERIDAE } \\
\hline Pinnixa spp. & Chuvoso & 2,27 \\
\hline \multicolumn{3}{|l|}{ OCYPODIDAE } \\
\hline Ucides cordatus (Linnaeus, 1763) & 2 picos: Seco e Chuvoso & 6,46 \\
\hline Uca spp. & Seco & 6,20 \\
\hline MAJIDAE & 2 picos: Seco e Chuvoso & 6,38 \\
\hline \multicolumn{3}{|l|}{ GRAPSIDAE } \\
\hline Armases angustipes (Dana, 1852) & Chuvoso & 3,06 \\
\hline Goniopsis cruentata (Latreille, 1803) & Seco & 1,62 \\
\hline Pachygrapsus gracilis (Saussure, 1858) & Seco & 5,58 \\
\hline Grapsidae morfotipo A & Seco & 1,89 \\
\hline \multicolumn{3}{|l|}{ GECARCINIDAE } \\
\hline Cardisoma guanhumi Latreille, 1825 & Chuvoso & 2,17 \\
\hline \multicolumn{3}{|l|}{$\begin{array}{l}\text { PORTUNIDAE } \\
\text { POR }\end{array}$} \\
\hline Callinectes spp. & Chuvoso & 7,93 \\
\hline
\end{tabular}

\section{DISCUSSÃO}

Na estação 1, o aporte de água doce, somado à precipitação pluviométrica, determinou uma baixa salinidade no período chuvoso, representado pelos meses de março a agosto. Na estação seca, de setembro a fevereiro, a concentração salina da água superou a concentração média da água do mar em ambas as estações, tornando-as hipersalinas. A circulação no interior do estuário do rio Jaguaribe é fortemente influenciada pela região marinha adjacente devido, entre outros fatores, à sua pequena vazão e ao processo de assoreamento em sua foz, que dificulta a troca de água na baixamar.

No presente estudo, não ocorreu variação bem definida de temperatura de acordo com a estação do ano (seca/chuvosa), principalmente na estação 2.

Cada crustáceo sobrevive dentro de uma determinada faixa de tolerância térmica, e, dentro dela, o aumento desta variável promove uma redução em seu desenvolvimento embrionário e larval. Este fator ambiental torna-se de grande importância, limitando a dispersão das zoeas em nível horizontal, além de promover uma redução deste período crítico, quando o animal é mais suscetível à predação (PINHEIRo et al., 1994). Além disso, a temperatura influencia a duração do período reprodutivo de algumas espécies de Brachyura exploradas comercialmente, como G. cruentata e U. cordatus (Botelho et al., 1999; Совo \& Fransozo, 2003).

Em termos de densidade, esta não diferiu entre as estações de coleta, e a elevada amplitude registrada na estação 1 parece ter relação com a grande variação da salinidade neste local (Fig. 2). Tanto a densidade (Fig. 4) quanto a salinidade foram mais baixas nos meses de maio a agosto, período correspondente à estação chuvosa.

Segundo GonZÁLEZ-GordiLlo \& RodRIGUEZ (2003), a ocorrência de baixa salinidade parece inibir ou dificultar o desenvolvimento larval de muitas espécies de decápodes. Experimentos de cultivo em laboratório evidenciaram que a salinidade igual ou próxima à do mar é ótima para o completo desenvolvimento larval de vários braquiúros (FRANSOZO, 1987; ANGER et al., 1990; LUPPI et al., 2003).

A ocorrência de uma maior densidade de zoeas no período noturno e na maré vazante, em ambas as estações de coleta, sustenta as hipóteses de comportamento antipredação e de exportação para áreas mais favoráveis. Nesse sentido, Luppi et al. (2003) destacaram duas estratégias para as larvas de espécies de decápodes, cujos adultos vivem em água salobra, doce, ou até em ambientes terrestres: retenção no habitat parental, ou exportação para condições mais estáveis em águas costeiras ou oceânicas.

A alternância do nível vertical na coluna d'água, em sincronia com o ciclo de marés, pode resultar tanto num transporte passivo a longas distâncias, como bloquear o transporte, ou seja, reter os indivíduos no local desejado e evitar o deslocamento para áreas menos favoráveis (GIBSON, 2003).

HAYs (2003) realizou uma revisão sobre o significado dos movimentos verticais dos organismos na coluna d'água, e destacou a hipótese de evasão contra predadores, a qual determina que a ascensão na coluna d'água durante o período noturno serve para reduzir o risco de predação por predadores visuais.

Na entrada da Baía de Guanabara, Estado do Rio de Janeiro, FERNANDES et al. (2002) registraram elevadas densidades de decápodes planctônicos, no verão e inverno, na superfície, durante a maré vazante noturna, e mais baixas nas marés enchentes diurnas. Da mesma forma, Silva et al. (2003) registraram maior densidade de larvas de decápodes em amostras noturnas do macrozooplâncton coletado no Canal de Santa Cruz, Estado de Pernambuco.

Embora não tenha ocorrido diferença significativa na densidade entre as estações de coleta, observou-se que, sazonalmente, houve uma diferença neste parâmetro, tendo as maiores densidades na estação 1 ocorrido no período seco, e no chuvoso na estação 2. Porém, na estação 2, os valores de densidade ao longo do ano foram 
mais homogêneos, indicando que a grande variação na densidade, observada na estação 1 , provavelmente foi decorrente da baixa salinidade registrada nesta estação, no período chuvoso.

FERnANDEs et al. (2002) afirmaram que, entre os decápodes planctônicos coletados na estação chuvosa na Baía de Guanabara, as zoeas de Brachyura foram o grupo mais importante, freqüentemente representando mais de $90 \%$ da densidade total de decápodes. Por outro lado, Silva et al. (2003) encontraram maior abundância destas larvas na estação seca, no Canal de Santa Cruz, onde, na estação chuvosa, predominaram os copépodes Calanoida.

Vale ressaltar que a abundância de zoeas é afetada pela época e duração do período reprodutivo, que varia de acordo com as condições geográficas e a composição faunística. A reprodução contínua é comum em braquiúros tropicais e subtropicais. Além disso, a dinâmica reprodutiva de uma espécie pode diferir em populações isoladas geograficamente (Reigada \& NegreirosFRANSOZO, 2000).

Ocypodidae constituiu o grupo dominante, sendo seus indivíduos freqüentemente coletados em amostras noturnas, principalmente na maré vazante, assim como Grapsidae, Majidae, Portunidae e a zoea morfotipo A. FERNANDES et al. (2002) constataram que a densidade de Ocypodidae, tanto no inverno como no verão, apresentava-se mais elevada na superfície em amostras noturnas. Porém, os autores registraram as famílias Portunidae a Grapsidae como mais abundantes, representando mais de $90 \%$ do total de zoeas.

As zoeas de Grapsidae, embora tenham apresentado um padrão de distribuição diferenciado entre as estações, foram registradas em quase todos os bimestres. Segundo Coвo \& Fransozo (2003), que avaliaram os fatores externos que determinam a estação de reprodução de G. cruentata na costa norte do Estado de São Paulo, a ocorrência de fêmeas ovígeras é quase constante durante todo o ano e consideraram este padrão comum entre as espécies de Grapsidae.

As zoeas de Portunidae ocorreram apenas na estação mais externa e no quarto bimestre; desta forma, não foi possível avaliar diferenças na densidade entre os fotoperíodos e marés. Na Lagoa da Conceição, Santa Catarina, o ciclo reprodutivo de Callinectes danae Smith, 1869 ocorre durante todo o ano, com alguns picos principais. Além disso, as fêmeas ovígeras migram através do canal que liga a lagoa ao mar, e a eclosão das larvas ocorre em águas mais salgadas ou no mar aberto (BRANCO $\&$ MASUNARI, 2000).

Não foi determinado um padrão de distribuição bem definido para as zoeas de Xanthidae, e sua densidade entre as marés e fotoperíodos não se apresentou diferenciada. Fernandes et al. (2002) também não encontraram um padrão para a distribuição das zoeas de Xanthidae, não registrando diferença significativa entre as estações seca e chuvosa.

De acordo com Negreiros-Fransozo (1986), Xanthidae constitui-se na mais numerosa família entre os braquiúros, e mesmo contando com um bom número de publicações sobre o desenvolvimento larval, a maioria das suas formas é ainda desconhecida. No presente estudo, foram registrados seis diferentes morfotipos de Xanthidae, os quais ocorreram em baixas densidades. Há indícios de que diferentes morfotipos podem constituir uma mesma espécie, já que existe a possibilidade da perda de estruturas imprescindíveis à identificação taxonômica, as quais podem ser facilmente danificadas durante a coleta do material.

As zoeas de Pinnotheridae, coletadas somente na maré enchente e dominantemente no período diurno, demonstraram preferir permanecer na área estuarina e foram mais abundantes na estação próxima à foz e no período chuvoso. Permanecer nas águas eutróficas estuarinas pode ser uma vantagem, ao se considerar a disponibilidade de alimento, porém é onde ocorrem altos níveis de poluição e altas taxas de predação (FERNANDES et al., 2002).

Por outro lado, Negreiros-Fransozo et al. (2002) enquadraram as zoeas de Pinnotheridae no grupo daquelas que realizam uma dispersão para as águas marinhas costeiras. Porém, é necessário considerar as condições ambientais ótimas para o desenvolvimento de cada grupo. Segundo GiBson (2003), podem existir diferenças regionais no comportamento de migração das larvas, em resposta às diferenças nos regimes de marés ou estrutura do habitat.

De uma maneira geral, foi observado um número maior de grupos que ocorrem preferencialmente na maré vazante, sendo transportados para as áreas externas do estuário, do que daqueles que predominaram na maré enchente, a qual permite uma retenção no estuário. SchwAmborn et al. (1999) constataram que cerca de $80 \%$ das zoeas que ocorrem na plataforma costeira adjacente à Ilha de Itamaracá eram derivadas das populações estuarinas.

O elevado número de espécies coletadas no primeiro estágio larval confirma o importante papel de berçário que as áreas estuarinas e costeiras desempenham (GonzÁlez-Gordillo \& Rodriguez, 2003). Pinnixa spp. (Pinnotheridae) foi o único táxon que apresentou todos os estágios larvais em Jaguaribe, indicando, mais uma vez, uma provável retenção nas águas estuarinas.

No estudo realizado por ScHWAMBORN et al. (1999) na região Nordeste, entre os Estados de Pernambuco e Ceará, foi registrada uma fraca dispersão das zoeas de Uca spp. e U. cordatus, onde as maiores densidades ocorreram próximo à enseada estuarina na Ilha de Itamaracá, ficando retidas nas plumas estuarinas durante a baixamar. A ocorrência de estágios larvais mais avançados de $U$. cordatus em ambas as estações sugere que outros fatores, além da migração vertical, podem ter influenciado o modo de distribuição deste grupo. NEGREIROs-FransOzo et al. (2002) supõem que as espécies que toleram uma ampla variação de salinidade são capazes de completar sua metamorfose dentro ou próximo ao estuário.

A colonização de áreas de berçário apropriadas é decisiva para a sobrevivência de pós-larvas e juvenis de muitas espécies de peixes e crustáceos (SCHWAMBORN \& BONECKER, 1996). Permanecer próximo à superfície durante a maré vazante pode resultar em rápida dispersão em direção ao oceano. Da mesma forma, permanecer no fundo pode resultar em baixa dispersão (FERNANDES et al., 2002). Apesar de não terem sido realizadas coletas de fundo, 
supõe-se que a pouca profundidade do Rio Jaguaribe, principalmente na estação à montante, pode ter mascarado a verdadeira distribuição de alguns indivíduos em algumas coletas.

Uma vez que foi observada uma maior influência da maré e do fotoperíodo, pode-se concluir que estes fatores promovem uma resposta comportamental às condições fisiológicas, já que houve uma variação quanto à distribuição das zoeas de Brachyura no estuário do Rio Jaguaribe.

\section{REFERÊNCIAS BIBLIOGRÁFICAS}

Anger, K.; Montú, M.; Bakker, C. \& Fernandes, L. L. 1990. Larva development of Uca thayeri Rathbun, 1900 (Decapoda:Ocypodidae) reared in the laboratory. Meeresforsch 32(1):276-294.

Botelho, E. R. O.; Dias, A. F. \& Ivo, C. T. C. 1999. Estudo sobre biologia do caranguejo-uçá, Ucides cordatus cordatus (Linnaeus, 1763), capturado nos estuários dos rios Formoso (Rio Formoso) e Ilhetas (Tamandaré), no Estado de Pernambuco. Boletim Técnico Científico do CEPENE 7(1):117-145.

Branco, J. O. \& Masunari, S. 2000. Reproductive ecology of the blue crab, Callinectes danae Smith, 1869 in the Conceição Lagoon System, Santa Catarina Isle, Brazil. Revista Brasileira de Biologia 60(1):17-27.

Coвo, V. J. \& Fransozo, A. 2003. External factors determining breeding season in the red mangrove crab Goniopsis cruentata (Latreille) (Crustacea, Brachyura, Grapsidae) on the São Paulo State northern coast, Brazil. Revista Brasileira de Zoologia 20(2): 213-237.

Ekau, W.; Westhaus-Ekau, P.; Macêdo, S. J. \& Dorrien, C. V. 2001. The larval fish fauna of the "Canal de Santa Cruz" estuary in Northeast Brazil. Tropical Oceanography 29(2):117-128.

Fernandes, L. D. A.; Bonecker, S. L. C. \& Valentin, J. L. 2002. Dynamic of decapod crustacean larvae on the entrance of Guanabara Bay. Brazilian Archives of Biology and Tecnology 45(4):491-498.

Fransozo, A. 1987. Desenvolvimento larval de Eriphia gonagra (Fabricius, 1781) (Decapoda, Xanthidae), em laboratório. Revista Brasileira de Zoologia 4(3):165-179.

Gibson, R. N. 2003. Go with the flow: tidal migration in marine animals. Hydrobiologia 503(1/3):153-161.

GonZÁlez-Gordillo, J. I. \& RodrigueZ, A. 2003. Comparative seasonal and spatial distribution of decapod larvae assemblages in three coastal zones off the south-western Iberian Peninsula. Acta Oecologica 24(1):219-233.

Grabe, S. A. 2003. Seasonal periodicity of decapod larvae and population dynamics of selected taxa in New Hampshire (USA) coastal waters. Journal of Plankton Research 25(4):417-428.

HAYs, G. C. 2003. A review of the adaptative significance and ecosystem consequences of zooplankton diel vertical migrations. Hydrobiologia 503(1/3):163-170.

Lacerda, S. R.; Koening, M. L.; Neumann-Leitão, S. \& Flores-Montes, M. J. 2004. Phytoplankton nyctemeral variation at a tropical river estuary (Itamaracá-Pernambuco-Brazil). Brazilian Journal of Biology 64(1):81-94.

Luppi, T. A.; SpIvak, E. D. \& Bas, C. C. 2003. The effects of temperature and salinity on larval development of Armases rubripes Rathbun, 1897 (Brachyura, Grapsoidea, Sesarmidae), and the southern limit of its geographical distribution. Estuarine, Coastal and Shelf Science 58(3):575-585.
Medeiros, C. \& KJerfve, B. 1993. Hydrology of a tropical estuarine system: Itamaracá-Brazil. Estuarine, Coastal and Shelf Science 36(1):496-515.

Melo, G. A. S. 1996. Manual de identificação dos Brachyura (caranguejos e siris) do litoral brasileiro. São Paulo, Plêiade/ FAPESP. 604p.

Negreiros-Fransozo, M. L. 1986. Desenvolvimento pós-embrionário de Panopeus americanus Saussure, 1857 (Decapoda, Xanthidae), em laboratório. Revista Brasileira de Biologia 46(1):173-188.

Negreiros-Fransozo, M. L.; Fransozo, A.; González-Gordillo, J. I. \& BertinI, G. 2002. First appraisal on releasing and reinvasion of decapod larvae in a subtropical estuary from Brazil. Acta Limnologica Brasiliensia 14(3):87-94.

Pinheiro, M. A. A.; Fransozo, A. \& Negreiros-Fransozo, M. L. 1994. Estimativa da duração larval em função da temperatura para a família Majidae (Crustacea, Decapoda, Brachyura). Boletim do Instituto de Pesca 21:75-81.

Pohle, G. W.; Mantellato, F. L. M.; Negreiros-Fransozo, M. L. \& Fransozo, A. 1999. Larval Decapoda (Brachyura). In: Boltovskoy, D. ed. South Atlantic Zooplankton. Leiden, Backhuys. v. 2, p. 1281-1351.

Reigada, A. L. D. \& Negreiros-Fransozo, M. L. 2000. Reprodutive cycle of Hepatus pudibundus (Herbst, 1785) (Crustacea, Decapoda, Calappidae) in Ubatuba, SP, Brazil. Revista Brasileira de Biologia 60(3):483-491.

SANDIFER, P. A. 1973. Distribution and abundance of decapod crustacean larvae in the York River Estuary and adjacent lower Chesapeake Bay, Virginia, 1968-1969. Chesapeake Science 14(4):235-257.

Schwamborn, R. \& Bonecker, A. C. T. 1996. Seasonal changes in the transport and distribution of meroplankton into a Brazilian estuary with emphasis on the importance of floating mangrove leaves. Arquivos de Biologia e Tecnologia 39(2):451-462.

Schwamborn, R.; Ekau, W.; Silva, A. P.; Silva, T. A. \& Saint-Paul, U. 1999. The contribution of estuarine decapod larvae to marine zooplankton communities in North-East Brazil. Archives of Fishery and Marine Research 47(2/3):167-182.

Schwamborn, R.; Neumann-Leitão, S.; Silva, T. A.; Silva, A. P.; Ekau, W.\& Saint-Paul, U. 2001. Distribution and dispersal of decapod crustacean larvae and other zooplankton in the Itamaracá estuarine system, Brazil. Tropical Oceanography 29(1):1-18.

Shanks, A. L.; Largier, J.; Brink, L.; Brubaker, J. \& Hooff, R. 2002. Observations of meroplankton during a downwelling event and associated intrusion of the Chesapeake Bay estuarine plume. Journal of Plankton Research 24(4):391-416.

Silva, T. A.; Neumann-Leitão, S.; Schwamborn, R.; Gusmão, L. M. O.\& NAscimento-VieiRA, D. A. 2003. Diel and seasonal changes in the macrozooplankton community of a tropical estuary in Northeastern Brazil. Revista Brasileira de Zoologia 20(3):439-446.

Silva, A. P.; Neumann-Leitão, S.; Schwamborn, R.; Gusmão, L. M. O. \& Silva, T. A. 2004. Mesozooplankton of an impacted bay in North Eastern Brazil. Brazilian Archives of Biology and Technology 47(3):485-493.

Vasconcelos-Filho, A. L. \& Oliveira, A. M. E. 1999. Composição e ecologia da ictiofauna do Canal de Santa Cruz (Itamaracá-PE, Brasil). Trabalhos Oceanográficos da UFPE 27(1):101-113.

Williams, A. B. 1971. A ten-year study of meroplankton in North Carolina estuaries: annual occurrence of some brachyuran developmental stages. Chesapeake Science 12(2):53-61.

Yannicelli, B.; Castro, L. R.; Valle-Levinson, A; Atkinson, L. \& FigueroA, D. 2006. Vertical distribution of decapod larvae in the entrance of an equatorward facing bay of central Chile: implications for transport. Journal of Plankton Research 28(1):19-37.

Recebido em maio de 2006. Aceito em abril de 2007. ISSN 0073-4721

Artigo disponível em: www.scielo.br/isz 